\title{
REDES SOCIALES PARA LA INTELIGENCIA COMPETITIVA. PROPUESTA DE UN ÍNDICE SINTÉTICO
}

Pilar González-Gálvez, Carina Rey-Martín y Víctor Cavaller-Reyes
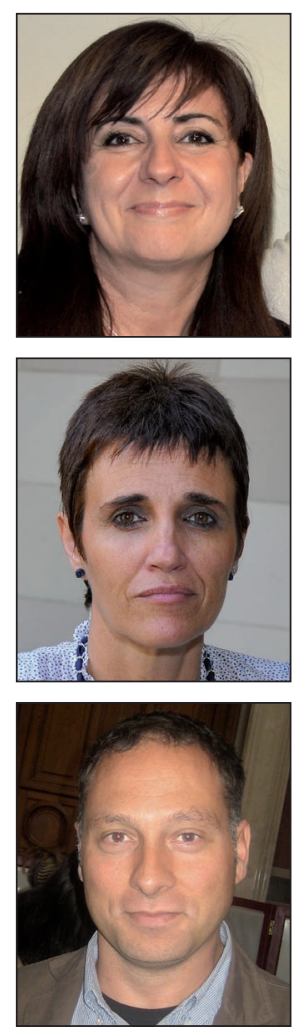

Víctor Cavaller-Reyes, doctor por la UB (2007) en el programa Informació i documentació en l'era digital del Departament de Biblioteconomia i Documentació. En el bienio 2002-2004 realizó la tesis: Sistema matricial d'indicadors per a l'anàlisi estratègica de la informació a les organitzacions. Licenciado en documentación (UOC, 2003) y en filosofía y ciencias de la educación (UB, 1992). Profesor de la UOC en los estudios de Ciencias de la Información y de la Comunicación y profesor asociado en el Departamento de Ciencias económicas y Empreseariales de la UIC.

vcavaller@uoc.edu

\section{Resumen}

Se presentan las bases teóricas y metodológicas de un modelo que ofrece al directivo información sobre el aprovechamiento que obtiene de su propia red social. Se analizan las redes sociales de los directores de enfermería de los hospitales de la Red Hospitalaria de Utilización Pública (XHUP) de Catalunya y se estudia la información que se recupera de dichas redes. Se aplica el modelo propuesto para realizar un análisis comparativo de los directivos estudiados, con el objetivo de iniciar estrategias de mejora de los resultados en los proyectos de inteligencia competitiva.

\section{Palabras clave}

Inteligencia competitiva, Red social, Recuperación de información, Análisis de redes sociales, Indicador sintético.

Title: Social networks that support competitive intelligence. Proposal of a synthetic index.

\section{Abstract}

We present the theoretical and methodological basis of a model that offers the managers information about their own social networks. We analyzed the nursing directors social networks of hospitals belonging to the Network of Hospitals for Public Use (XHUP) of Catalonia, Spain, and studied the information retrieved from these networks. The proposed model is applied to make a comparative analysis of managers surveyed, in order to improve strategies and to enhance outcomes of competitive intelligence projects.

\section{Keywords}

Competitive intelligence, Social networks, Information retrieval, Social network analysis, Synthetic indicator.

González-Gálvez, Pilar; Rey-Martín, Carina; Cavaller-Reyes, Víctor. "Redes sociales para la inteligencia competitiva. Propuesta de un índice sintético". El profesional de la información, 2011, septiembre-octubre, v. 20, n. 5, pp. 527-532. http://dx.doi.org/10.3145/epi.2011.sep.06

Artículo recibido el 01-07-11

Aceptación definitiva: 13-09-11 


\section{Introducción}

Una parte de la información que utilizan los directivos para llevar a cabo las funciones de inteligencia propias de su cargo, proviene de su red social (Cross; Prusak, 2002; Cetisme, 2003; Tena; Comai, 2004). Usamos el concepto de red social en el más amplio sentido de la expresión sin limitarnos al uso que se está haciendo actualmente con las plataformas de social networking. La expresión "red social" referida a estas plataformas, ha pasado a los medios de comunicación y a nuestro lenguaje cotidiano, de manera que se usan de manera sinónima (Leiva, 2009; Leiva, 2010; Roca, 2010; Molina, 2011). En el presente trabajo se considera el concepto de red social desde una dimensión basada en la antropología social. Y a pesar de estar de acuerdo en que internet proporciona una valiosa potencia a las formaciones reticulares, no se puede obviar que en nuestro día a día usamos también relaciones que no están sustentadas en la tecnología.

En un contexto de cambios constantes, en que las organizaciones deben ser más competitivas y buscar la excelencia, resulta imprescindible tener un conocimiento exhaustivo del entorno. Uno de los instrumentos empleados en inteligencia competitiva para obtener información de valor estratégico lo constituye la red de relaciones y contactos de la organización y sus integrantes. Dicha información está vinculada al concepto de capital social (Brunet; Belzunegui, 2001; Ortoll et al., 2008).

Las redes sociales son un canal de transmisión de conocimiento, experiencias, información, datos, consejos, etc., muy valorados por los directivos. A pesar de que esta vía de acceso a la información es reconocida de manera tácita, generalmente las organizaciones no suelen contemplarla como una parte formal del ciclo de inteligencia y por lo tanto, no se plantean acciones estratégicas para explotarla o mejorarla (Cross; Prusak, 2002).

La primera condición para iniciar una mejora es conocer la situación inicial, y posteriormente establecer una comparativa con las posiciones ocupadas por otras organizaciones. Para ello elegiremos unos parámetros para evaluar el rendimiento que el directivo obtiene de su red social.

\section{Objetivos}

Presentamos un modelo que incluye un indicador sintético llamado Índice de la Función de Inteligencia Competitiva (IFIC). Se trata de un indicador compuesto que integra diversos indicadores simples que permiten comparar el comportamiento de los directivos.

Dicho modelo forma parte de una investigación en curso, cuyos objetivos y marco teórico son más extensos, y que pretende conocer en qué medida las redes sociales de los directores de enfermería de los hospitales de la Red Hospitalaria de Utilización Pública de Catalunya (XHUP), constituyen un instrumento de soporte a las funciones de inteligencia de los mismos. No profundizaremos en el estudio marco pero serán necesarias algunas referencias al mismo, puesto que la elaboración del IFIC parte de una exhaustiva revisión bibliográfica y de una rigurosa metodología llevadas a cabo en el estudio inicial, sin las cuales el modelo presentado no se podría sustentar.

\section{Metodología}

Para la elaboración del presente estudio se realiza una combinación de investigación cualitativa-cuantitativa, sincrónica paralela coordinada (Smith, 1985).

La parte cualitativa, sustentada en una serie de entrevistas a expertos en redes sociales, inteligencia competitiva y comunicación empresarial, nos ha permitido elegir los atributos necesarios para conformar el IFIC. Se ha optado por un muestreo intencional opinático (Vallés, 2002), donde los informantes se eligen en base a un criterio estratégico personal. En este caso, su conocimiento de la situación a investigar es lo que los hace representativos. En cuanto al número de entrevistas realizadas (12) se ha seguido un proceso de acumulación de entrevistas (Vela-Peón, 2008) hasta conseguir un "punto de saturación" en que se han alcanzado los puntos de interés y los resultados de nuevas entrevistas no aportan ya información relevante para la investigación.

La parte cuantitativa está basada en un cuestionario enviado por correo electrónico, elaborado expresamente para esta investigación con el propósito de estudiar la tipología de la red social de los directores de enfermería de la XHUP y analizar la información que procede de dichas redes. Para reducir el error de no respuesta (total y de ítem) hemos seguido el Total design method (Dillman, 2000), que incrementa la tasa de respuesta. La recogida de datos estandarizada y sistematizada ha permitido la comparación entre los distintos directivos estudiados.

Los errores de especificación, de medida, de respuesta y los posibles sesgos de la encuesta se han minimizado siguiendo un riguroso proceso de planificación de la encuesta. Para evitar los errores de especificación, se ha llevado a cabo una cuidadosa definición de los conceptos clave en relación con las medidas del problema de investigación. Para ello se han seleccionado dichos conceptos, identificando los constructos derivados de los mismos (Hox, 1997). Seguidamente, en una fase de operacionalización, estos constructos teóricos se han pasado a variables observables. En base a los objetivos de la investigación, se han utilizado estas variables derivadas del concepto clave, para medir la actitud relacional de los directores de enfermería. Así se ha llegado a concretar una relación de atributos mesurables, que presenta coherencia con el concepto clave y por tanto, se han evitado los errores de especificación. Para controlar los errores de medida y de respuesta, se han considerado aspectos como la formulación de las preguntas, el contexto de las mismas, las opciones de respuesta, la redacción y la vía de administración del cuestionario. Hemos querido así asegurar que todos los encuestados pudieran hacer idéntica interpretación de las preguntas (Cea, 2004).

Antes de iniciar los envíos de la encuesta se procedió a pasar un pre-test para validar el cuestionario y comprobar su funcionamiento.

Se dirige la encuesta al universo de estudio, es decir, a la totalidad de unidades de las que deseamos obtener información. Se trata pues de un análisis censal (Casal; Mateu, 2003) que quiere estudiar la población de los directores/as de enfermería de todos los hospitales pertenecientes a la $X H U P$, entre abril y julio de 2010 . El hecho de que la mues- 
tra sea relativamente pequeña $(n=61)$ permite un mayor control de la investigación. Por otro lado, examinar a toda la población posibilita conocer exactamente la distribución que presentan las variables estudiadas en esta población (Casal; Mateu, 2003). El marco muestral es el directorio de los hospitales de la XHUP (Decreto 124/2008, de 25 de junio, DOGC n오 5.161).

\begin{tabular}{|l|l|}
\hline \multicolumn{2}{|c|}{ Ficha técnica del cuestionario } \\
\hline $\begin{array}{l}\text { Población objeto de } \\
\text { estudio }\end{array}$ & $\begin{array}{l}\text { Directores/as de enfermería de los hospitales } \\
\text { de la XHUP. Se trata de 61 individuos } \\
\text { (68 centros) }\end{array}$ \\
\hline Unidad de análisis & Individuos \\
\hline $\begin{array}{l}\text { Método de recogida } \\
\text { de la información }\end{array}$ & $\begin{array}{l}\text { Cuestionarios enviados vía electrónica con } \\
\text { una carta de presentación del estudio }\end{array}$ \\
\hline $\begin{array}{l}\text { Duración del trabajo } \\
\text { de campo }\end{array}$ & Abril-julio 2010 \\
\hline $\begin{array}{l}\text { Tamaño de la } \\
\text { muestra }\end{array}$ & $\begin{array}{l}48 \text { cuestionarios. 13 cuestionarios no } \\
\text { recibidos }\end{array}$ \\
\hline $\begin{array}{l}\text { Tasa de respuesta } \\
\text { efectiva }\end{array}$ & $78,7 \%$ \\
\hline $\begin{array}{l}\text { Tratamiento de la } \\
\text { información }\end{array}$ & Rebost de dades v.4.0 (U.B.) y SPSS v.18.0 \\
\hline
\end{tabular}

\section{Presentación del modelo}

Con la propuesta del IFIC se ha pretendido condensar la información contenida en una serie de atributos o indicadores sobre determinados aspectos relacionales de los directivos. Se trata de una medida sintética del uso que dichos directivos hacen de sus redes sociales, de cara a obtener la información más adecuada para dar soporte a sus funciones de inteligencia competitiva. En este sentido se contemplan diversos aspectos relativos a su red de contactos y también a las características de la información que circula por ella.

Somos conscientes de que la utilización de un índice sintético, comporta cierto grado de subjetividad, en cuanto a las decisiones que toma el investigador, como la selección, la normalización, la ponderación y la agrupación de los atributos (Blancas et al., 2009). Sin embargo, a pesar de que dicha subjetividad puede influir en los resultados obtenidos (Nardo et al., 2005), los índices sintéticos se consideran adecuados como instrumentos de medición analítica en ciencias sociales (Blancas; Contreras; Ramírez, 2011).

La metodología usada para la obtención de los indicadores es participativa (Domínguez et al., 2011), es decir que considera las valoraciones subjetivas de individuos de referencia, respecto a los aspectos que se quieren analizar.

La información útil de la red social de los directores de enfermería para la toma de decisiones es compleja de medir, tanto por los elementos que la conforman, como para la obtención de unos resultados comprensibles y útiles para establecer categorías. Es justamente por esta complejidad que a partir de los atributos iniciales se determina un conjunto de indicadores simples que ofrecen un resumen de la información sobre la función de inteligencia competitiva en las dos dimensiones básicas determinadas: la red social del directivo y la información que éste recupera de la misma. Cada una de estas dimensiones (dimensión red y dimensión información) están conformadas por varios factores o indicadores simples, que ofrecen una aproximación sobre determinados aspectos. Estos indicadores son, para la dimensión red: la estructura, la morfología y las interacciones que tienen lugar en la misma. Y para la dimensión información: funciones de inteligencia, tipo de información que circula y calidad de la misma.

A la vez estos indicadores simples presentan diferentes atributos que son los que se han medido a través de los apartados del cuestionario. Para la dimensión red, los atributos explorados son: centralidad, betweenness (intermediación), cohesión, rango, duración de las relaciones, direccionalidad de los contactos y frecuencia con que se mantienen. Para la dimensión información, los atributos son: valoración que los directivos hacen de la efectividad de la misma, rentabilidad, tipo de información, tipo de fuente, fiabilidad de la información, relevancia, suficiencia y grado de actualización.

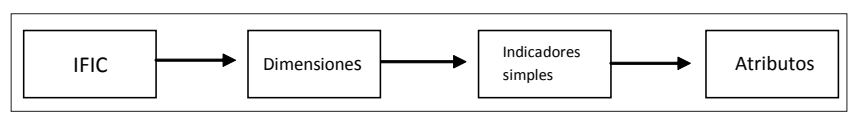

A partir de la revisión bibliográfica llevada a cabo en el estudio que enmarca el presente artículo y las entrevistas a expertos, se han elegido los atributos más adecuados para conformar el IFIC. Seguidamente presentamos una breve definición de cada uno de ellos.

Dimensión red:

- Centralidad: posición o localización del actor (director de enfermería) en el conjunto de toda la red, que determina su grado de acción. Un individuo es central si está bien conectado con los otros de su entorno (Freeman, 1979).

- Betweenness: depende de la posición relativa de cada actor y mide el grado de conexiones que pasan por él para que un actor llegue a otro (Freeman, 1991).

- Cohesión: informa de la existencia de vínculos entre los actores y mide la conciencia colectiva (Alonso, 2006).

- Rango: potencial de acción de los actores (Lozares, 1996), cuáles son los que tienen acceso directo a otros actores.

- Duración: período de vida de las relaciones sociales. El objetivo de explorar este atributo es comprobar si influye en la información aportada por las relaciones.

- Direccionalidad: observa si la dirección de la interacción repercute en la influencia de un actor hacia otro.

- Frecuencia: repetición de los contactos entre los actores.

Dimensión información:

- Valoración de la efectividad: considerando que efectividad es la capacidad de producir el efecto deseado, en este caso sería la obtención de información útil a partir de las conversaciones mantenidas con los miembros de la red social.

- Rendimiento: beneficio obtenido de los medios utilizados, o sea la información obtenida a través de los contactos.

- Tipo de información: por una parte se valora el tipo de información que proporciona cada tipo de relación, y por otra, se compara la red interna y la externa, analizando el tipo de información que proviene de cada una de ellas (operativa, legal, estratégica, técnica, política, económica).

- Tipo de fuentes. 
- Fiabilidad de las fuentes: el valor que una información tiene para un directivo es difícilmente mesurable dada la fuerte carga subjetiva que acompaña al concepto valor. Por ello nos hemos fijado en aspectos como la fiabilidad o credibilidad de la fuente.

- Relevancia: idoneidad respecto a la necesidad planteada.

- Suficiencia: información que contiene todos los datos importantes y no precisa ser completada.

- Actualización: vigencia de la información recibida.

Estos parámetros se exploran mediante un cuestionario creado expresamente para esta investigación que contiene 28 items, distribuidos en 4 bloques. El primero incluye preguntas sobre la red de relaciones. En él se exploran aspectos como la estructura, la morfología y la interacción de la red. La información derivada de este primer bloque se ha utilizado para el desarrollo del IFIC, concretamente para los cálculos de los atributos que conforman la dimensión red.

El segundo bloque contiene preguntas referidas a la información que circula por la red. Se exploran las características en cuanto a tipo y calidad. Los resultados de estas cuestiones se han utilizado para los cálculos de los atributos de la dimensión información del IFIC.

El tercer bloque está conformado por preguntas sobre el uso que los directores hacen de la información que les llega a través de su red social. La información resultante se ha usado también para los cálculos de la dimensión información.

Finalmente, el cuarto bloque, contiene cuatro preguntas sobre datos de los directores o del centro donde desarrollan su función profesional.

El IFIC se calcula como la suma aritmética del valor que toma cada uno de los atributos citados. Se ha optado por otorgar un peso equitativo para cada uno de los atributos analizados. Asimismo, para cada uno de ellos se ha tomado un recorrido de 0 a 1 determinado de manera proporcional según la cantidad de respuestas posibles. De este modo el resultado del IFIC sintetiza el valor de cada uno de los 15 atributos elegidos. Finalmente, el valor del IFIC, cuyo recorrido va de 0 a 15, se ha normalizado, obteniendo así, valores que van de 0 a 1 .

La tabla 1 muestra la descomposición del IFIC en sus dos dimensiones: red e información, y la división de las mismas en indicadores simples y atributos.

\begin{tabular}{|c|c|c|c|c|c|c|c|c|c|c|c|c|c|c|c|}
\hline Indicador & \multicolumn{15}{|c|}{ IFIC } \\
\hline $\begin{array}{l}\text { Dimensio- } \\
\text { nes }\end{array}$ & \multicolumn{7}{|c|}{ Red } & \multicolumn{8}{|c|}{ Información } \\
\hline $\begin{array}{l}\text { Indicadores } \\
\text { simples }\end{array}$ & & שِّ & & 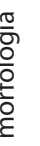 & & 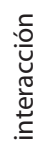 & & 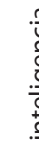 & $\frac{\sigma}{\frac{\sigma}{\nu}}$ & & & \multicolumn{4}{|c|}{$\begin{array}{l}\frac{0}{\pi} \\
\frac{\pi}{0} \\
\frac{0}{0}\end{array}$} \\
\hline Atributos & 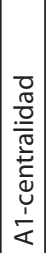 & 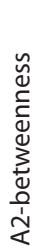 & 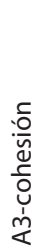 & $\begin{array}{l}\stackrel{\circ}{O} \\
\frac{c}{0} \\
\frac{1}{3} \\
\frac{1}{4}\end{array}$ & 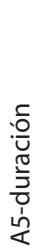 & 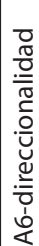 & 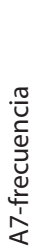 & 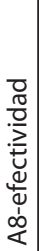 & 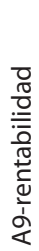 & 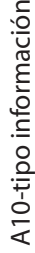 & 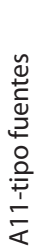 & 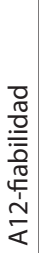 & 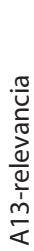 & 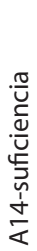 & 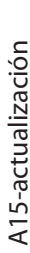 \\
\hline
\end{tabular}

Tabla 1. Estructura del IFIC
En primer lugar se han definido el valor y el recorrido de cada atributo para determinar en qué manera participan en la valoración de los indicadores simples.

Ello va a permitir evaluar la red de cada uno de los directores y verificar si los que disponen de una mejor posición en la dimensión red, obtienen a su vez una mejor posición en la dimensión información.

El valor mínimo de IFIC (IFIC $=0$ ) se produce cuando se observa la ausencia de red de relaciones y de información obtenida. A medida que aumenta el grado de relaciones y/o la presencia de información en la red del directivo, el indicador toma valores positivos crecientes hasta un máximo de 1 .

\section{Resultados}

En función de la valoración y los recorridos de estos 15 atributos, se genera una hoja de cálculo que proporciona el valor de los indicadores simples, de las dimensiones red/información y consecuentemente el valor del IFIC para cada uno de los 48 directivos estudiados. Con tales valores se ha construido el gráfico 1 . En el eje horizontal se sitúan los valores normalizados de la dimensión Red (constituida por sus siete atributos descritos), y en el eje vertical los valores normalizados de la dimensión Información (constituida por sus ocho atributos). El tamaño de la burbuja se corresponde al valor del IFIC normalizado.

Los directivos quedan distribuidos en cuatro cuadrantes:

A: dimensión red baja- dimensión información alta

B: dimensión red alta- dimensión información alta

C: dimensión red baja- dimensión información baja

D: dimensión red alta- dimensión información baja

En el cuadrante A (red baja-información alta) se encuentran los directivos que utilizan poco su red social y a pesar de ello obtienen una cifra elevada de información de la misma. Sólo son 4 casos (8\%).

Analizando alguno de ellos ( $n$ - 30, con un IFIC $=0,64$ ) se observa que el nivel bajo de la dimensión red $(0,55)$ se debe sobre todo a la morfología de la misma, puesto que el atributo cohesión presenta un valor nulo. En cuanto al nivel alto de la dimensión información $(0,72)$ se explica sobre todo por la máxima valoración de la efectividad que el directivo hace del uso de dicha información y por la calidad de la misma, en especial por su máximo nivel de actualización. Asimismo se observa una baja puntuación respecto al tipo de información que se obtiene de la red y también respecto al tipo de las fuentes consultadas.

En el cuadrante B (red alta-información alta) se encuentran los directivos que utilizan su red social de manera óptima y obtienen de ella una buena información. Se observa que no se trata de un cuadrante muy numeroso: 9 casos (19\%).

Analizando el mejor de los casos ( $n=27$, con un IFIC = 0,90) se observa que el elevado nivel de la dimensión red $(0,86)$ se debe a la estructura de la misma (centralidad elevada; máximo nivel de betweenness) y también a su morfología (cohesión máxima; rango elevado). Respecto a la dimensión información $(0,94)$ se aprecia una alta puntuación de la capacidad para hacer un uso inteligente de la misma (efectividad y rentabilidad máximas), así como unos niveles máxi- 
mos para los indicadores de calidad de la información (fiabilidad; relevancia; suficiencia; actualización).

En el cuadrante $\mathbf{C}$ (red baja-información baja) se hallan los directivos que menos utilizan su red social y que obtienen poca información o, en cualquier caso, les es de escasa utilidad para tomar de decisiones. Es un grupo más numeroso que los dos anteriores: 12 casos (25\%).

Analizando el caso con puntuación más baja ( $n$ o 31 , con un IFIC $=0,48$ ) se observa que el bajo nivel de la dimensión red $(0,54)$ se explica mayormente por la morfología de la misma (cohesión nula; rango bajo), aunque el resto de indicadores de esta dimensión tampoco pre-

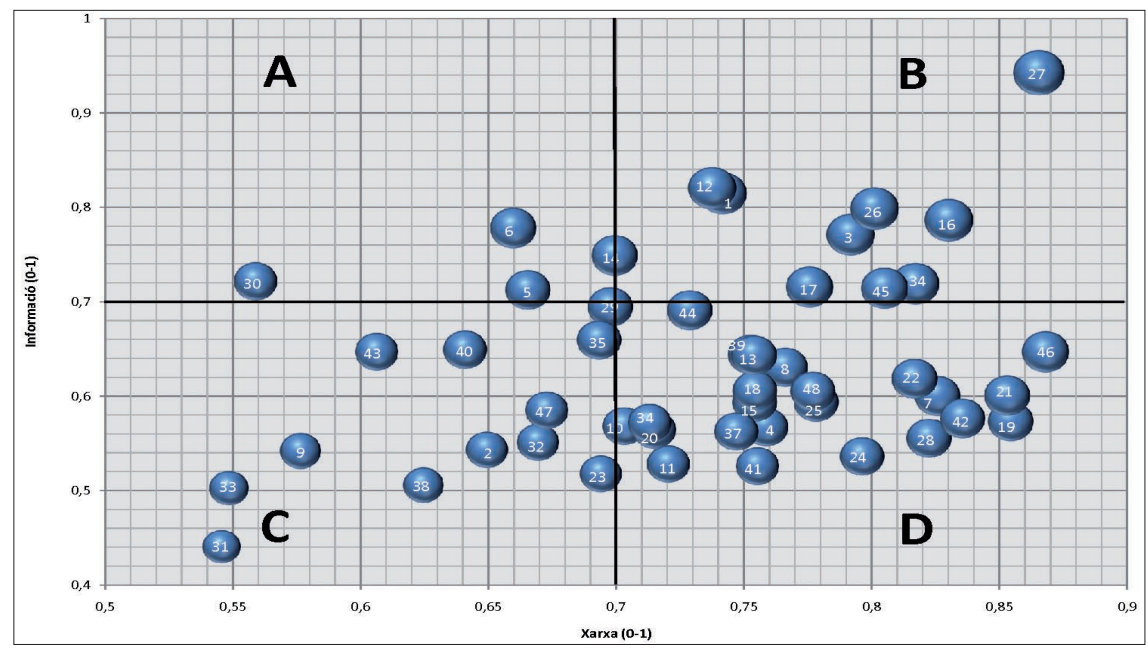

Gráfico 1. Posicionamiento de los directivos estudiados respecto a las coordenadas dimensión información y dimensión red. sentan puntuaciones muy buenas y por supuesto, ninguno de los atributos alcanza el 1. Respecto a la dimensión información $(0,44)$ apreciamos las peores puntuaciones respecto a los tipos de información y de las fuentes consultadas. El resto de indicadores apenas alcanzan valores cercanos a 0,5.

En el cuadrante $\mathbf{D}$ (red alta-información baja) se encuentran los directivos que a pesar de tener una buena cifra en la dimensión red, no obtienen de su red social una información óptima para dar soporte a su toma de decisiones. Es el cuadrante que contiene una mayor representación de directores: 23 casos (48\%).

Analizando el caso no 46 (IFIC $=0,75$ ) que muestra la puntuación más alta en dimensión red de este cuadrante $(0,86)$ se observa que responde a unos buenos niveles de estructura, morfología e interacción. Destacan los niveles máximos de betweenness, cohesión y duración de las relaciones. Respecto a la dimensión información $(0,64)$ se observa una bajísima puntuación en los atributos relacionados con los tipos de información y de fuentes. Respecto al uso inteligente que el directivo puede hacer con dicha información se constatan valores relativamente bajos.

\section{Conclusiones}

- Se observa una muy escasa presencia de directores en el grupo A, que presenta baja puntuación en la dimensión red y obtiene alta puntuación en la dimensión información. Nos parece lógico que se trate de un grupo poco numeroso y se propone fortalecer los atributos con menores puntuaciones para conseguir mejorar el posicionamiento hacia el grupo $B$.

- El cuadrante C, integrado por directores con las peores puntuaciones, responde a una relación directa: baja puntuación en red y baja puntuación en información. Parece evidente que los directivos pertenecientes a este grupo no han tomado conciencia de la importancia de explotar su red de relaciones y por lo tanto, no prestan atención a la información que podrían obtener de la misma.

- Los directores que dedican esfuerzos a optimizar su red social (mitad derecha del gráfico) no siempre obtienen la mejor información. El grupo B integra a los que sí obtienen los mejores resultados en la dimensión información, a resultas de estar bien posicionados en la dimensión red. Se observa que existen directores con puntuaciones red similares, que se hallan en el grupo D (alta puntuación en red-baja información). En muchos de los casos no están utilizando el tipo de fuentes adecuado. Posiblemente variar las fuentes consultadas o perseguir otros tipos de información les haría mejorar su puntuación en la dimensión información.

- Consideramos que el uso de este indicador puede resultar de utilidad en otros colectivos. En cualquier caso, la principal ventaja de utilizar el IFIC es poder averiguar cuáles son los atributos peor puntuados para aplicar en ellos las medidas de mejora correspondientes. Si se consigue usar este indicador de forma generalizada podremos llevar a cabo comparaciones entre directivos y optar a los mejores rendimientos.

\section{Referencias bibliográficas}

Alonso, Luis-Enrique. "Centralidad del trabajo y cohesión social: una valoración necesaria". Gaceta sindical: reflexión y debate, 2006, n. 7, pp. 101-126. ISSN: 1133-035X.

http://www.unavarra.es/puresoc/pdfs/c_salaconfe/SCAlonso-Trabajo.PDF

Blancas-Peral, Francisco-Javier, et al. "El indicador sintético DCP como instrumento de medición de la sostenibilidad turística". XVII Jornadas Asepuma, Burgos, 2009, actas, n. 17. http://www.uv.es/asepuma/XVII/112.pdf

Blancas-Peral, Francisco-Javier; Contreras-Rubio, Ignacio; Ramírez-Hurtado, José M. "Construcción de indicadores sintéticos: una aproximación para maximizar la discriminación". XVII Jornadas Asepuma, Valencia, 2011, actas, n. 19. http://www.uv.es/asepuma2011/comunicaciones/0110.pdf

Brunet, Ignasi; Belzunegui, Ángel. "En torno a las redes de empresa y territorio". Revista española de investigaciones sociológicas, 2001, n. 95. pp. 69-98.

Casal, Jordi; Mateu, Enric. "Tipos de muestreo". Revista epidem. med. prev., 2003, n. 1, pp. 3-7.

Cea-D’Ancona, María-Ángeles. Métodos de encuesta. Teoría y práctica, errores y mejora. Madrid: Ed. Síntesis, 2004, 493 pp. 
Cetisme. Inteligencia económica y tecnológica. Guía para principiantes y profesionales. Madrid: Innovación, Desarrollo y Transferencia Tecnológica SA (coord), 2003, 128 pp. ISBN: 84-451-2390-4.

Cross, Rob; Prusak, Laurence. "Redes informales: identifique a las personas clave". Harvard Deusto business review, 2002, n. 110, pp. 50-60.

Denzin, Norman K. The research act: A theoretical introduction to sociological methods. $2^{\text {nd }}$ ed. New York: Mc Graw-Hill, 1978. ISBN: 0070163618

Dillman, Don A. Mail and internet surveys: the tailored design method. New York: Wiley, 2000.

Domínguez-Serrano, Mónica; Blancas-Peral, Francisco-Javier; Guerrero-Casas, Flor-María; González Lozano, Mercedes. "Una revision para la construcción de indicadores sintéticos". Revista de métodos cuantitativos para la economía y la empresa, 2011, n. 11, pp. 41-70.

http://redalyc.uaemex. $m x /$ redalyc/pdf/2331/2331183 02004.pdf

DOGC no 5161. Decret 124/2008, de 25 de juny.

Freeman, Linton C. "Centrality in social networks. Conceptual clarification", Social networks, 1979, n. 1, pp. 215-239.

Freeman, Linton C.; Borgatti, Stephen P.; White, Douglas R. "Centrality in valued graphs: A measure of betweenness base don network flow", Social networks, 1991, n. 13, pp. 141-154.

Hox, Joop J. "From theoretical concept to survey questions". In: Lyberg, L. [et al]. Survey measurement and process quality. New York: John Wiley and Sons Inc., 1997, pp. 47-70.

Leiva-Aguilera, Javier. "Informe redes sociales". Baratz, 2009.
http://www.slideshare.net/Baratz/informe-redes-socialesjavier-leiva

Leiva-Aguilera, Javier. Entrevista con los autores. Vic, 17/06/10.

Lozares, Carlos. "La teoría de las redes sociales". Papers, 1996, n. 48, pp. 103-126.

Molina, José-Luis. Entrevista con los autores. Bellaterra, 15/02/11.

Nardo, Michaela et al. Tools for composite indicadors building. Institute for the Protection and Security of the Citizen. European Commission, 2005.

Ortoll, Eva et al. "El capital social com a font d'intel-ligència competitiva a les universitats". UOC Papers, n. 7, 2008. ISSN: 1885-1541

http://www.uoc.edu/uocpapers/7/dt/cat/ortoll_lopez_ cobarsi_garcia_canals.pdf

Roca, Genís. Entrevista con los autores. Barcelona, 01/07/10

Smith, Robert B. "Linking quality quantity". En: Smith, R. A handbook of social science methods. New York: Praeger, 1985.

Tena, Joaquín; Comai, Alessandro. La inteligencia competitiva en las mejores prácticas españolas, Barcelona: PuzzleEmecom, 2004. ISBN: 8460936864

Vallés, Miguel S. Entrevistas cualitativas. Madrid: Centro de Investigaciones Sociológicas. Cuadernos metodológicos, 32, 2002. ISBN: 9788474763423

Vela-Peón, Fortino. "Un acto metodológico básico de la investigación social: la entrevista cualitativa”. En: Tarrés, M. L. (coord.) Observar, escuchar y comprender. Sobre la tradición cualitativa en la investigación social. México: El Colegio de México, pp. 408, 2008. ISBN: 9789707012028

\section{Si te interesan los}

\section{INDICADORES EN GIENGIA Y T G NOLOGÍA,}

y todos los temas relacionados con la mediaien de la ciencia, tales como:

Análisis de citas, Normallz-ieich de la ciencia en la sociedad, indieren so volo de ciencia, Política científica, Comunicación de tere o hele Revistas, Bases de datos, Índices de impacto, Politicas de open ccess, A tial sis de la nueva economía, Mujer y ciencia, etc.

\section{Entonces INCYT es tu lista. Suscribete en:}

